\title{
Extending Lifespan of the Network using Shortest Path Low Latency Routing Protocol (SPLLR) for Wireless Sensor Networks
}

\author{
D.S.Bhargava, T.Blesslin Sheeba' T.V.Padmavathy, S.Jagadeesh Babu
}

Keywords: Wireless Sensor Networks (WSN), Energy Efficiency, Throughput, Latency.

\section{INTRODUCTION}

Sensor networks have of a more number of sensors, processing circuits, and a wireless transceiver [11],[12],[14]. The size of a sensor node is small in order to in remote and unmanned areas. When the nodes are deployed, they organized themselves as a network, and it can monitor the data in the application area. The nodes get the energy from the battery cell, since the size of a cell is limited; the amount of available energy is also limited. Therefore, in sensor networks the reduction of energy used by the each sensor nodes is the major issues. Lifetime of the sensor networks is mostly depends on consumption of energy in each node during transmission and reveal of data [13]. In energy optimization technique takes priority in WSNs for continuous transmission of data thereby enhancing the network lifetime. By considering above cons proper design has to make for each sensor node so that the efficiency of networks can be improved.

In order to reduce the energy usage in each sensor nodes and to extend the lifetime of sensor networks an energy management protocols and techniques have to be addressed.

Revised Manuscript Received on September 27, 2019

* Correspondence Author

D.S. Bhargava, Assistant professor in ECE in R.M.K Engineering College, Mail: dsb.ece@rmkec.ac.in

Dr.T.Blesslin Sheeba, Professor, Department of ECE in R.M.K Engineering College, Mail: tbs.ece@rmkec.ac.in

T. V. Padmavathy, Professor, Department of ECE in R.M.K. Engineering College, Mail: tvp.ece@rmkec.ac.in

S.Jagadeesh Babu, Assistant Professor, Department of ECE in R.M.K. Engineering College, Mail: sjb.ece@rmkec.ac.in

\begin{abstract}
Energy Management is the one of the key factor in Wireless Sensor Networks (WSN) in order to improve the network lifetime. Though broadcasting of information with minimum energy is key concern in wireless sensor networks, it often introduces an additional delay. Existing network protocols has pros and cons interms of energy consumption and various performance metrics. Therefore most of the existing protocols are not suitable for continuous transfer and receiving of data from sensor nodes. A novel protocol is proposed for solving the existing issues in the algorithms. In this protocol we use a quadratic assignment technique to design an energy efficient protocol. This proposed protocol provides better efficiency in terms of energy, throughput and reduced end to end latency during transmission and reception
\end{abstract}

There is no rule to choose a particular architecture for monitoring data in any applications. Wireless Sensor Nodes formed a cluster of densely deployed nodes to communicate the sensed information through sink nodes. Most of the sensor networks constrained by the low power, dense deployed and limited processing speed. Wireless Sensor Networks has small, cheap, independent, and disposable sensor nodes.

\section{RELATED WORKS}

Usually the sensor nodes Deployed in the unmanned areas and with the limited battery capacity. Therefore the lifetime of sensor nodes is a major issue. There are several techniques are proposed in the recent year [1-6] The routing protocols of wireless sensor networks are classified based on the topology, data centric, location assisted and mobility based. Data Collection EDAL protocol for heterogeneous network was proposed in [7]. Here they used data collection based on energy level of each node and it gives higher performance in all aspects but it is not suitable for large a network that is scalability is poor. Genetic based algorithm and Greedy based Genetic based concepts was proposed in [8]. In this paper they addressed the $\mathrm{k}$ connectivity algorithm to find the residual energy. This algorithm is suitable only for number of intermediate node is less. Vehicles assisted data delivery protocols was proposed in [10]. This protocol is used to forward the packet to the best path. But this protocol suffers low data delivery. Phase Wise data aggregation protocol was presented in [9]. This protocol minimizes the data collection from nodes.

\section{MATHEMATICAL MODEL FOR SPLLR}

Let $C_{i j}$ be the wireless sensor network with set of $\mathrm{N}$ nodes $N=\{1,2,3, \ldots \ldots, n\}$. Let $S_{i}$ represent the set of one hop neighbor node $i \in N$.

$e_{(a v g) i, j}^{t}$ be the average energy consumed for data transmission from node $i$ to node $j$

$E_{i}$ be the initial battery energy level of node $i$

$S$ be the set of source nodes which is deployed in the application area, where the $i^{\text {th }}$ node senses the data with rate of $Q_{i}$ 


\section{Extending Lifespan of the Network using Shortest Path Low Latency Routing Protocol (SPLLR) for Wireless}

Sensor Networks

$D$ be the set of destination nodes, which is received the data from the source node.

$\gamma_{i j}$ be the traffic generated at node $i$ due to the neighbor node $j$,

$\alpha_{i j}$ be the amount of data that needs to be transmitted from node $i$ to node $j$

The proposed approach uses optimal path algorithm in order to reduce the energy usage of sensor node during transmission and reception. The Quadratic Assignment Problem can be defined by three $n \times n$ matrices and it is represented by,

$F=\left(f_{i j}\right), D=\left(d_{i j}\right), C=\left(c_{i j}\right)$,

where $F, D$ and $C$ are coefficients of QAP. In this approach we have to find a permutation $\rho$ with set of $\mathrm{N}$ nodes $N=\{1,2,3, \ldots \ldots, n\}$, which sensor will transfer the data to the destination. Based on the permutation the quadratic functional can be written as,

$T(\rho)=\sum_{i=1}^{n} \sum_{j=1}^{n} f_{i j} d_{\rho(i) \rho(j)}$

Permutation matrix $X=\left\lfloor x_{i j}\right\rfloor$ is formed for every permutation $\rho$, The permutation matrix can be written as,

$\sum_{i=1}^{n} x_{i j}=1 \quad$ for $j=1$ to $n$

$\sum_{j=1}^{n} x_{i j}=1 \quad$ for $i=1$ to $n$

Where $x_{i j} \in\{0,1\}$ for $i, j=\{1,2, \ldots \ldots, n\}$. The above equation is also known as permutations constraints. Therefore the QAP can be written as

$$
x_{i j}= \begin{cases}1 & i^{\text {th }} \text { node condition is satified } \\ 0 & \text { Otherwise }\end{cases}
$$

$\arg \min _{X} T=\arg \min _{X} \sum_{i, j=1}^{n} \sum_{k l}^{n} f_{i k} d_{j l} c_{k l} x_{i j}$

The above equation (4) is satisfied only if the energy and buffer level are within threshold then only the $i^{\text {th }}$ node will transmit the data to the $j^{\text {th }}$ node.

\section{PROPOSED PROTOCOL SPLLR}

A novel algorithm for route selection in WSN can be designed based on the equation (4) which is called as Shortest Path Low Latency Routing Protocol (SPLLR), the key of improvement in our route selection algorithm is that the performance of SPLLR protocol can be achieved by selecting the recent route to the intended destination. The novel algorithm is known as NSRSA (New Shortest Route
Selection Algorithm). It uses the new-short path as routing metric. NSRS algorithm estimates the shortest new of the route. Based on the route reply packet $\left(R_{r e p}\right)$ from source a new route is selected for transferring of data from $i^{\text {th }}$ node to $j^{\text {th }}$ node. In common, standard existing protocol, $R_{\text {rep }}$ packets answer back by intermediate nodes or destination node. Intermediate nodes use their route cache to send $\left(R_{r e p}\right)$ packet to the source node. Fig 1 and 2 shows the packet format for route reply packet $\left(R_{r e p}\right)$ and route request packet $\left(R_{\text {req }}\right)$ respectively.

\begin{tabular}{|c|c|c|c|c|c|}
\hline $\begin{array}{c}\text { Message } \\
\text { Type }\end{array}$ & $\begin{array}{c}\text { Sende } \\
\mathrm{r} \text { ID }\end{array}$ & $\begin{array}{c}\text { Node } \\
\text { Type }\end{array}$ & $\begin{array}{c}\text { Hop } \\
\text { Coun } \\
\mathrm{t}\end{array}$ & $\begin{array}{c}\text { Forwar } \\
\mathrm{d} \\
\text { Node ID }\end{array}$ & $\begin{array}{c}\text { Forward } \\
\text { Node } \\
\text { Energy } \\
\text { Level }\end{array}$ \\
\hline
\end{tabular}

Fig. 1 Route Reply Packet $\left(R_{r e p}\right)$

\begin{tabular}{|c|c|c|c|c|c|}
\hline $\begin{array}{c}\text { Message } \\
\text { Type }\end{array}$ & $\begin{array}{c}\text { Sende } \\
\mathrm{r} \text { ID }\end{array}$ & $\begin{array}{c}\text { Sin } \\
\mathrm{k} \text { ID }\end{array}$ & $\begin{array}{c}\text { Route } \\
\text { ID }\end{array}$ & $\begin{array}{c}\text { Lin } \\
\mathrm{k} \\
\text { Cost }\end{array}$ & $\begin{array}{c}\text { Forward } \\
\text { Node Energy } \\
\text { Level }\end{array}$ \\
\hline
\end{tabular}

Fig. 2 Route Request Packet $\left(R_{r e p}\right)$

When the nods are deployed in dynamic scenario then if the mobility of nodes is high then there will be discontinue of path will occur. In this case, route selection process becomes a time consuming process if the communicating route fails frequently.

QAA method gives the priority for $R_{\text {rep }}$ packet which is generated from destination node rather than intermediate nodes. If there is more than one $R_{\text {rep }}$ packet answer, back from the destination node, then the shortest-new route will be selected. Whereas, if there is no $R_{\text {rep }}$ packet response from the destination node, the source node will select the shortest route in its route cache regardless of the source of $R_{\text {rep }}$ packet. Basically, QAA algorithm has five steps during routing process, which will be described as the following:

\section{A. STEPS INVOLVED IN QAA}

Step 1: If the node wants to send data packets to a destination node:

i. If the source's cache has one or more routes; it selects the shortest route based on the acknowledgement signal received from the destination node that is the priority gives for routes which replied by the destination.

ii. If the source's cache has not a route to the destination; the source node propagates route request packet $\left(R_{r e q}\right)$, and wait for route reply packet $\left(R_{r e p}\right)$.

Step 2: If an intermediate node receives a $R_{r e q}$ : 
i. If it has a path to the destination, send $R_{r e p}$ with Flag =

False to the source.

ii. If has not; re-propagates $R_{r e q}$.

Step 3: If the destination node receives a $R_{\text {req }}$ :

i. Send $R_{\text {rep }}$ with Flag=True towards the source.

ii. Stop the propagating of $R_{r e q}$.

Step 4: If an intermediate node receives a new $R_{\text {req }}$ : Caches the route with its flag status (False/True). Forward the $R_{r e q}$ packet to the next intend node towards the source.

Step 5: when the source node receives a $R_{\text {req }}$, caches the route, then check the cache:

i. If there is one or more than $R_{r e q}$ with Flag=True, select the recent-short one.

ii. If there is no $R_{r e q}$ with Flag=True, select the shortest one.

Stop the propagating of $R_{r e q}$.

\section{B. Shortest Path Algorithm}

Let us consider $N$ numbers of homogeneous sensor nodes are deployed randomly in the application area. At any time, a sensor node $n$ is able to extract the reaming energy (residual energy) level $E_{n-\text { residual }}$ of its battery. Whenever a node is detecting the event, the neighbouring nodes exchange the data between them. Based signal strength received from the node, select one of the nodes as the source node. The source node has the responsibility to collect the data from the neighboring nodes and to transmit the collected data to the sink node. Within a coverage area if the different event occurs then the data from the nodes are collected and not being transmitted to the sink node. A link may contain $k$ number of intermediate nodes which will transfer the data from $i^{\text {th }}$ node to $j^{\text {th }}$ node until it reaches the destination. Here we assumed that a network is dense so there will be multiple links is exist from $i^{\text {th }}$ node to $j^{\text {th }}$ node. We assume that the multiple links used are disjoint. The length of the link that is number of hop count between $i^{t h}$ node to $j^{\text {th }}$ node as named as cost which is used to find out the neighbouring nodes. Let $N_{x}$ be the set of neighbouring nodes $x$ in the link. Selection of next hop from the set of neighbouring nodes $x$ by using following condition.

$\arg \min _{y \in N_{x}}\left\{\left(\frac{1-E_{y-\text { residual }}}{E_{y \text {-initial }}}\right)^{\beta\left(1-\frac{\Delta d+1}{d_{x j}}\right)}\right\}$

Where $d_{x j}$ is the distance in hops between set of neighbouring node $x$ and $\operatorname{sink} j$; $d_{y j}$ is the distance in hops between set of neighbouring node $y$ and $\operatorname{sink} j$;

$\Delta d$ is the distance between $d_{x j}$ and $d_{y j}$;

$E_{y \text {-initial }}$ and $E_{y-\text { residual }}$ be the initial and residual energy of node $y$;

$\alpha \quad$ is the weight factor and $\alpha>1$.

Note that $(\Delta d+1) \varepsilon\{0,1,2\}$ and $\left(\frac{1-E_{y \text {-residual }}}{E_{y \text {-initial }}}\right) \varepsilon(0,1)$.

To form the link between transmitting nodes $i$ and sink node $j$, we have to consider the energy level of neighbouring node and the hop count.

In case the residual energy of neighbouring node $y$ is constant then the link cost get increases because the lifetime of the link get increases. If $(\Delta d+1)$ remains constant then the link cost increases. In this case, the link cost increases and $E_{y \text {-residual }}$ get reduced. The link cost of the node is given by, $\sum_{k=i}^{k=j}(i+1)$

(6)

\section{SIMULATION RESULT ANALYSIS}

After the running the proposed protocol with these environmental based tool, we have come up with the results in terms of energy consumption, Control Message Overhead, Average Data Transfer delay, Packet Delivery Ratio. Also we compare our proposed algorithm with the already existing routing protocol such as DD, Flooding, energy aware and multi path routing.

\section{A. Average energy consumption Vs Node Count}

Average energy usage is refers to the energy consumed in average by every node present in the network. Fig 3 , shows the average energy consumption of sensor node based on the number of nodes. This plot infers that the energy utilization of SPLLR routing is stable and has little impact by the increase of the network size, while the performance of other schemes degrades with larger network size. Due to the proposed shortest path algorithm the energy consumption of sensor node is reduced irrespective of the network size compared to the existing protocol. Compared to energy aware routing protocol $13 \%$ of energy and $94.4 \%$ is saved in SPLLR protocol when the number of node is 20 and 100 respectively. 


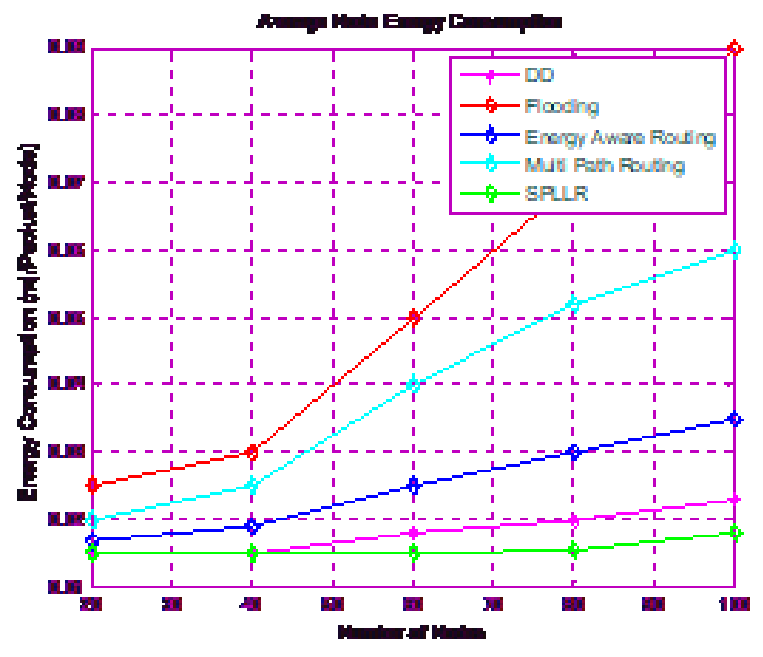

Fig.4 Control Message Overhead Vs Node Count

\section{B. Control Message Overhead Vs Node Count}

Fig 4 shows the control message overhead of different protocols with respect to number of nodes. It is obtained by calculating the ratio between the average amount of control message processed by the node and the number of data packets received by the sinks. Since the direct diffusion routing protocol requires periodic broadcasting of message to the neighbouring nodes for link reinforcement. Therefore the directed diffusion protocol exhibits more energy on transmitting and receiving the control messages than any other protocols. The proposed SPLLR protocol has lower overhead for control message so it consumes less energy compared to other protocols. Compared to energy aware protocol SPLLR routing protocol saves $95 \%$ and $87.5 \%$ for 100 and 20 nodes respectively.

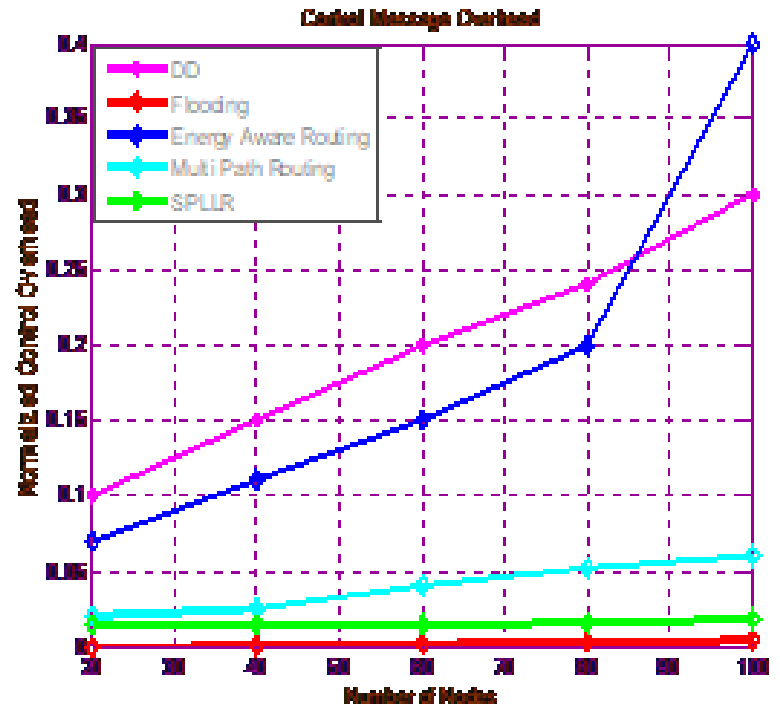

Fig. 5 Average Data Transfer Delay Vs Node Count

\section{Average Data Transfer Delay Vs Node Count}

Fig 5 shows the average data transfer delay between various protocols. Data transfer delay is determined by the channel bandwidth, packet length and the coding scheme adopted. The SPLLR routing protocol has the shortest delay compared to other schemes. As we expected, data packets are routed through different node-disjoint paths with shortest path energy efficient routing. Hence, the network congestion can be avoided.

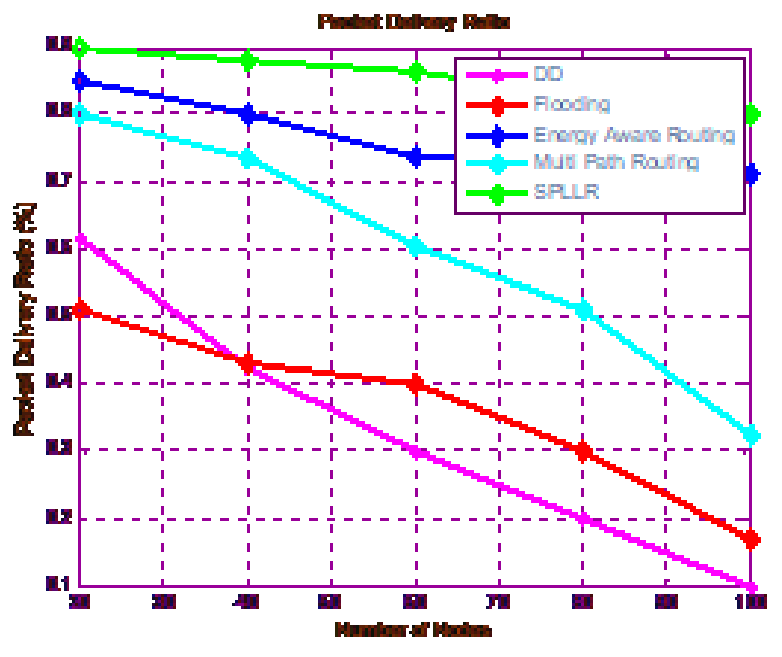

Fig.6 Packet Delivery Ratio Vs Node Count

\section{Packet Delivery Ratio Vs Node Count}

Packet delivery ratio is between ratios of acknowledgement packet received from the $j^{\text {th }}$ node to the total number of packets sent from $i^{\text {th }}$ node. Fig 6 shows the percentage packets delivered in each round based on number of nodes. It is to be noted that SPLLR protocol consistently gives higher percentage of packets delivered in comparison to direct diffusion and flooding protocol.

From the Fig 6, SPLLR outperforms than the DD and flooding because of limited congestion due to less routing overhead. It is to be noted that more than $42.15 \%$ and $30.28 \%$ of packets delivered in SPLLR routing protocol compared to Flooding and DD for 20 nodes and $84.67 \%$ and $71.07 \%$ of packets delivered in SPLLR routing protocol compared to Flooding and DD for 100 nodes. The ratio of data packets delivered to the destination and the data packets generated by the CBR sources are taken as packet delivery.

\section{CONCLuSION}

In this paper, the proposed a Shortest Path Energy Efficient Routing protocol (SPLLR) for wireless sensor networks is capable to search multiple node-disjoint links. The New Shortest Route Selection algorithm aims to allocate the traffic rate to each link optimally. From the simulation results we infer that the SPLLR scheme has higher node energy efficiency, lower average delay and control overhead. In this paper we considered well known protocols for wireless sensor networks called DD, Flooding, Energy aware and multipath routing for comparison to emphasis the efficiency of our proposed algorithm. Finally the data packet delivery in SPLLR routing protocol is more than that using DD, flooding, energy aware and multipath routing. But in this protocol we are not considering the performance parameters such as Fault tolerance and scalability of the sensor network In future the work may extend to improve the interns of data aggregation and scalability. 


\section{REFERENCES}

1. S. Soro and W. B. Heinzelman, "Cluster head election techniques for coverage preservation in wireless sensor networks," Ad Hoc Networks, vol. 7, no. 5, pp. 955-972, 2009.

2. C. Wang, J. Li, Y. Yang, and F. Ye, "Combining solar energy harvesting with wireless charging for hybrid wireless sensor networks," IEEE Transactions on Mobile Computing, vol. 17, no.3, pp. 560-576, 2017.

3. G. Anastasi, M. Conti, M. Di Francesco, and A. Passarella, "Energy conservation in wireless sensor networks: a survey," AdHoc Networks, vol. 7, no. 3, pp. 537-568, 2009.

4. I. F. Akyildiz, W. Su, Y. Sankarasubramaniam, and E. Cayirci, "Wireless sensor networks: a survey," Computer Networks, vol.38, no. 4, pp. 393-422, 2002.

5. W. K. G. Seah, Z. A. Eu, and H.-P. Tan, "Wireless sensor networks powered by ambient energy harvesting (WSN-HEAP) - survey and challenges," in Proceedings of the 1st International Conference on Wireless Communication, Vehicular Technology, Information Theory and Aerospace \& Electronic Systems Technology (Wireless VITAE '09), pp. 1-5, Aalborg, Denmark, May 2009.

6. L. J. Chien, M. Drieberg, P. Sebastian, and L. H. Hiung, "A simple solar energy harvester for wireless sensor networks," in Proceedings of the 6th International Conference on Intelligent and Advanced Systems (ICIAS'16), pp. 1-6, August 2016.

7. Y. Yao, Q. Cao, and A. V. Vasilakos, "EDAL: An energy-efficient, delay-aware, and lifetime-balancing data collection protocol for heterogeneous wireless sensor networks," IEEE/ACM Transactions on Networking (TON), vol. 23, no. 3, pp. 810-823, 2015.

8. S. K. Gupta, P. Kuila, and P. K. Jana, "Genetic algorithm for kconnected relay node placement in wireless sensor networks," in Proceedings of the second international conference on computer and communication technologies. Springer, 2016, pp. 721-729.

9. H. Harb, A. Makhoul, D. Laiymani, and A. Jaber, "A distancebased data aggregation technique for periodic sensor networks," ACM Transactions on Sensor Networks (TOSN), vol. 13, no. 4, p. 32, 2017.

10. Hassan Oudani, SalahddineKrit, Mustapha Kabrane, Kaoutar Bandaoud, Mohamed Elaskri, Khaoula Karimi, Hicham Elbousty, Lahoucine Elmaimouni, “ Energy Efficient in Wireless Sensor Networks Using Cluster-Based Approach Routing”, International Journal of Sensors and Sensor Networks 2017; 5(5-1): 6-12.

11. Shrestha, Akhilesh Xing, Liudong, "A Performance Comparison of Different Topologies for Wireless Sensor Networks", IEEE Conference on Technologies for Homeland Security, May 2007.

12. M. Rausand and A. Hoyland, "System Reliability Theory: Models and Statistical Methods", Wiley Series in Probability and Mathematical Statistics, John Wiley \&Sons, 2004

13. Dang Quang and Hwang Won-Joo,"Trade-off between Reliability and Energy Consumption in Transport Protocols for Wireless Sensor Networks", International Journal of Computer Science and Network Security, Vol.6 No.8B, August 2006.

14. Wang, X.; Xing, G. Integrated coverage and connectivity configuration in Wireless Sensor Networks. Proceedings of ACM International Conference on Embedded Networked Sensor Systems 2003, pp 28-39.

\section{AUTHORS PROFILE}

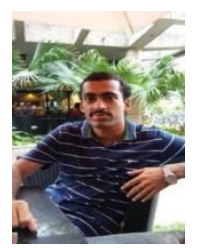

D.S. Bhargava Assistant professor in Electronics and Communication Engineering department of R.M.K Engineering College has 3 years of teaching experience. He received his Bachelor's degree from J.N.N Institute of Engineering in Electronics and Communication Engineering in the year 2012 and Master's degree in VLSI Design from R.M.K Engineering College in the year 2014 His area of interests includes Cognitive radio networks and VLSI Design technology. He has published 7 research papers in International and National Journals and conferences in the area of VLSI Design, Networking and Cognitive radio networks. He is a Life member of ISTE.

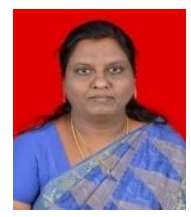

Dr.T.Blesslin Sheeba received her Ph.D in Crytptographic Algorithms for Small Embedded Applications from Sathyabama University, Chennai. She is working as Professor in the department of ECE, RMK Engineering College, Chennai. She has 26 years of Teaching and research experience. She has published more than 25 research papers in International and National Journals. Her current area of research includes security and architecture issues in networks and Antenna design. She is recognized as Fellowship member by The Institution of Engineers (India) also she is a member of various professional bodies such as
Life member of Indian Society for Technical Education ISTE, IACST, ACM and ISSE.

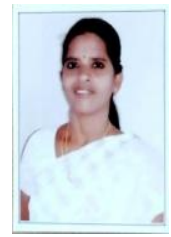

T. V. Padmavathy Professor, Department of ECE in R.M.K Engineering College, has 24 years of teaching and research experience in the in the fields of Wireless sensor networks, Under Water Acoustic Sensor Networks and Antenna Design. She has graduated from Institution of Engineers (India), in Electronics and Communication Engineering. She has obtained her Master degree in Control and Instrumentation from College of Engineering, Guindy, Anna University, Chennai and Ph.D. degree from Anna University, Chennai. She has published more than 50 research papers in International and National Journals and conferences in the area of Mobile Ad hoc Networks, Wireless sensor networks, Under Water Acoustic Sensor Networks and Antenna design and she has four Patents in Wireless Sensor Networks. Her current area of research includes security and architecture issues of Mobile ad hoc networks, Wireless sensor networks and Millimeter Wave Antenna design for Wireless Communications. She is a technical paper reviewer for African Journal of Engineering Research and Journal of Engineering and Technology Management. She is recognized as Fellowship member by The Institution of Engineers (India) also she is a member of various professional bodies such as Institute of Electrical and Electronics Engineers (IEEE), Life member of Institution of Electronics and Telecommunication Engineers (IETE), International Association of Engineers (IAENG), ACM, ISSE and Life member of Indian Society for Technical Education ISTE.

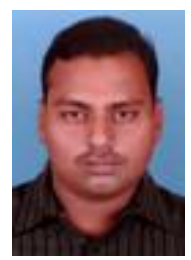

S.Jagadeesh Babu, Assistant Professor, Department of ECE in R.M.K. Engineering College, has 10 years of teaching and research experience in the in the fields of VLSI Design, Wireless sensor networks and Wireless Communication. He has graduated from Adhiparasakthi Engineering College, in Electronics and Communication Engineering. He has obtained his Master degree in VLSI Design from Easwari Engineering, Chennai. His current area of research includes Low power VLSI and proficient in backend tool CADENCE. He is a member of various professional bodies such as Life member of Institution of Electronics and Telecommunication Engineers (IETE), International Association of Engineers (IAENG), and Life member of Indian Society for Technical Education ISTE. 